Boise State University

ScholarWorks

$2-1-2018$

\title{
Education for WIC Peer Counselors About Breastfeeding the Late Preterm Infant
}

Cindi Faith Bennett

St Luke's Health System

Cynthia Galloway

Central District Health Department

Jane S. Grassley

Boise State University

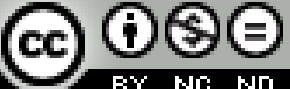




\section{EDUCATION FOR WIC PEER COUNSELORS ABOUT BREASTFEEDING THE LATE PRETERM INFANT \\ INTRODUCTION}

Approximately 7\% of all infants in the United States are born between 34 and 36

completed weeks gestation. Categorized as late preterm, they comprise $71 \%$ of all preterm births. ${ }^{1}$ Although breastfeeding has benefits that are particularly important for this vulnerable population, large cohort studies consistently demonstrate that breastfeeding initiation, duration, and exclusivity rates for infants born before 37 weeks are significantly lower than for those born at term. ${ }^{2}$ Late preterm infants demonstrate feeding behaviors that differ from full-term infants. ${ }^{3,4}$ Their immature suck and sleepiness can lead to delayed lactogenesis and an insufficient milk intake, placing them at risk for poor weight gain, dehydration, and jaundice and increasing the likelihood of hospital readmission in the first 2 weeks. ${ }^{3,4}$

Mothers who want to exclusively breastfeed may find themselves unprepared for the challenges presented by their developmentally immature infants. ${ }^{5,6}$ These mothers and infants often transition from hospital to home with a complex feeding plan that involves first latching the infant for a limited time using a nipple shield, then offering some expressed human milk or infant formula, and following-up with milk expression using a breast pump. ${ }^{4}$ Mothers often find this feeding regimen to be overwhelming and difficult to manage. ${ }^{5,6}$ On-going breastfeeding support after discharge, both professional and peer, is essential for this vulnerable population of mothers and infants. ${ }^{5,6}$

The Special Supplemental Nutrition Program for Women, Infants, and Children (WIC) breastfeeding peer counselors can play an important role in providing needed social support for mothers breastfeeding a late preterm infant. ${ }^{7}$ Evidence suggests that contact with a WIC peer counselor facilitates women's decisions to initiate and continue breastfeeding. ${ }^{7}$ Peer counselors 
1 are WIC clients who have been recruited and hired to provide breastfeeding support to current

2 clients. ${ }^{7,8}$ They have breastfed at least one child and ideally are the same racial/ethnic

3 background as the mothers they support. ${ }^{8}$ They offer basic breastfeeding information,

4 encouragement, and emotional support to mothers via phone, text, clinic visits, hospital and/or

5 home visits and are available outside of normal WIC clinic hours. ${ }^{7,8}$ Their role is to reinforce

6 breastfeeding recommendations and promote the benefits of breastfeeding for mothers and

7 children. They work with a WIC designated breastfeeding expert who assists with breastfeeding

8 management problems that are outside their scope of practice. ${ }^{8}$

$9 \quad$ WIC peer counselors receive specialized training through the Loving Support program

10 about best practices related to healthy term infants. ${ }^{8}$ However, late preterm infants, due to their

11 immaturity, need different evidence-based practices to establish breastfeeding. ${ }^{4,9}$ Understanding

12 these differences can help peer counselors offer more effective support to their clients. The

13 purpose of this report is to present information about breastfeeding and late preterm infants that

14 can supplement the Loving Support $₫$ peer counseling curricula currently used to educate WIC

15 peer counselors. $^{8}$

16

17

18

19

\section{DISCUSSION OF EDUCATION}

The following content was developed to facilitate WIC peer counselors’ knowledge of breastfeeding the late preterm infant. Topics discussed include characteristics of the late preterm infant and benefits of human milk; typical infant behaviors that affect breastfeeding; lactation technologies that help establish a milk supply that supports infant growth; mothers' experiences, and warning signs that need follow-up.

\section{Characteristics of the Late Preterm Infant}


During the final 4 to 6 weeks of pregnancy, fetuses experience a critical period of rapid brain growth and maturation of organ and body systems to ready them for life outside the womb. Glycogen stores increase in their livers; subcutaneous tissue and brown fat stores develop; muscle tone increases, and passage of maternal antibodies through the placenta increases. ${ }^{4,8,9}$ The brain experiences significant growth during this period with a 5-fold increase in white matter and $33 \%$ growth in brain volume. ${ }^{10}$ Figure 1 depicts this rapid brain growth in the final weeks of pregnancy. ${ }^{10}$ These changes prepare newborns for breastfeeding, which requires stamina, an ability to regulate sleep/awake cycles, and a coordinated suck and swallow. ${ }^{4,9,11,12}$

Human milk has properties that benefit these immature newborns. ${ }^{9,12}$ For example, the composition of their mothers' milk is specific to their needs as late preterm infants. Nutritional and anti-infective components are more highly concentrated in their milk and it is easier to digest. The milk contains higher levels of nitrogen, protein, sodium, chloride, and lipids (which also provides long-chain poly unsaturated fatty acids), all of which aid in the development of an infant's brain and nervous system. ${ }^{4,9}$ Human milk also promotes the development and maturation of the healthy intestinal microbiome essential to life-long digestive health. ${ }^{13}$

Human milk and breastfeeding are important to the future health of late preterm infants. Unfortunately, their immaturity influences their ability to effectively breastfeed. Because they have difficulty staying awake and coordinating their suck and swallow, their milk intake may be insufficient to support growth. ${ }^{4,9,12}$ These behaviors can interfere with lactogenesis II or copious milk production. Occurring 2 to 3 days’ post birth, this delay is often caused by lack of nutritive sucking that is needed to stimulate the prolactin hormone and feedback inhibitor of lactation, affecting milk production. ${ }^{12,14}$ These immature breastfeeding behaviors often result in a cascade of events that ends in hospital readmission for dehydration or jaundice (See Figure 2). By better 
understanding typical behaviors of late preterm infants, WIC breastfeeding peer counselors can provide mothers with important informational and emotional support.

\section{Typical Late Preterm Infant Behaviors}

Typical newborn behaviors exhibited by late preterm infants can affect breastfeeding outcomes. Understanding their feeding cues can be complicated by differences in infant gestation, development, and individual characteristics. ${ }^{5}$ In the early postpartum period they may act like full term infants and appear to be breastfeeding well during the short time they are in the hospital. ${ }^{4}$ However, their immaturity predisposes them to behaviors that affect their ability to effectively breastfeed, such as excessive sleepiness and short feedings. ${ }^{3-5,9,15,16}$ High energy demands and low energy stores decrease their feeding stamina, resulting in short feedings due to fatigue rather than being satiated..$^{3-5,9,16,17}$ This insufficient milk intake interacts with decreased alert, awake periods. ${ }^{4,11,15-17}$ Parents often struggle to make sense of their infant's behavior ${ }^{5,6,10}$ and may interpret these behaviors as signs of effective breastfeeding. ${ }^{4}$ Their immaturity impacts their ability to suck effectively. ${ }^{12,15,18}$ Late preterm infants typically exhibit low muscle tone and underdeveloped cheek pads, which affects their ability to maintain sufficient intra-oral pressure; as a consequence, they slip on and off their mother's nipple during feedings. ${ }^{5,6,18}$ These behaviors contribute to a disorganized nonnutritive sucking pattern that affects their ability to transfer an adequate amount of milk from the breast. ${ }^{12,17}$ These normal late preterm behaviors contribute to suboptimal breastfeeding, characterized by an insufficient amount of nourishment for infant growth and maternal difficulty with milk supply., ${ }^{4,17}$

\section{Use of Lactation Technologies}

Continued use of a hospital grade pump, nipple shields, and a baby weight scale become a way of life until the infant reaches 40 to 44 weeks gestational age and is working toward 
exclusive breastfeeding. ${ }^{4}$ The three goals for managing breastfeeding in this transition are "protecting milk volume, ensuring adequate milk intake during breastfeeding, and facilitating milk intake.” Milk volume is best protected by continued use of a hospital grade breast pump until the infant is exclusively breastfeeding. ${ }^{9,19,20}$ A hospital grade breast pump is used to mimic the breastfeeding patterns of a healthy full term infant and stimulate milk production. The pump is set at the maximum level of comfort with no pain; the mother's nipple should be centered in a correctly fitted breast shield and should move easily with very little areola in the flange tunnel when pumping. ${ }^{4}$ WIC peer counselors can offer important emotional support to mothers in the arduous task of pumping. ${ }^{6}$ Mothers need to understand the importance of routinely using the hospital grade breast pump and gradually discontinuing pumping when their infants are exclusively breastfeeding to ensure an adequate milk supply., ${ }^{49,20}$ Mothers who have difficulties can be referred to a certified lactation consultant at their WIC clinic or infant's birth hospital. ${ }^{8}$ Nipple shields can effectively facilitate milk intake during breastfeeding. ${ }^{4,9,18}$ Adequate milk consumption is a challenge because late preterm infants have difficulty maintaining an effective latch and decreased stamina and alertness. ${ }^{5,11}$ Nipple shields, which are available in different sizes, create an intra-oral vacuum that help the infant remain latched and transfer milk from the breast. ${ }^{4,18}$ Mothers need reassurance that use of the nipple shield is temporary and reminded to not prematurely discontinue its use. ${ }^{4,18}$ Early supplementation is advised to ensure adequate growth until the late preterm infant can exclusively breastfeed. ${ }^{4,9}$ The hospital discharge feeding plan often includes triple feeding, which can involve following breastfeedings with a supplement of expressed human milk or infant formula. ${ }^{9,21}$ However, it is difficult to estimate how much the infant needs because usual indicators like audible swallows and satiety are inaccurate for late preterm infants. ${ }^{4,21-23}$ Meier et al. ${ }^{4}$ strongly recommend that parents have 
access to an in-home baby scale to accurately measure intake and guide supplementation

needed. ${ }^{4,9,22-23}$ Mothers may find the day to day work of pumping and using a nipple shield to be a burden. ${ }^{5,6,21}$ By understanding the rationale for using these tools, WIC peer counselors can encourage mothers to persevere until their infant is effectively breastfeeding.

\section{Mothers' Experiences}

Mothers who are breastfeeding a late preterm infant experience feelings of uncertainty, anxiety, failure and exhaustion. They feel unprepared for their infants' feeding difficulties and the intense work of breastfeeding. ${ }^{5,6.11,24,25}$ Health care providers often minimalize the impact of gestation and fail to provide mothers with sufficient information and support about potential problems. 5,6,11,22,24 This collision of reality with expectations can cause parental stress; mothers may not meet their goal to exclusively breastfeed, which can increase their risk for depression and a delay in bonding with their infant. ${ }^{5,25}$ Informed emotional support is crucial. ${ }^{5,6}$

\section{Potential Warning Flags}

WIC breastfeeding peer counselors may be the first providers to recognize warning signs that warrant a call by the mother to the infant's healthcare provider. For example, excessive sleepiness while at breast or a yellow skin color can be indicators that the infant is not receiving enough human milk. ${ }^{26}$ Other red flags include having less than 6 wet diapers and 2 yellow stools per day after day 5 and a lethargic infant who is difficult to awaken for feedings. ${ }^{26}$ To identify potential breastfeeding problems and facilitate early intervention, the American Academy of Breastfeeding Medicine ${ }^{26}$ recommends that "the late preterm infant should have weekly weight checks until 40 weeks post conceptual age or until he or she is thriving."

The following questions can be a helpful guide to the mother's perceptions about breastfeeding her infant. How does your infant look? Is there anything that looks different to 
1 you? Does your infant stay awake while eating? Or do you have to coax him/her to stay awake through the feeding? How long does a feeding take? How are you feeling about breastfeeding? How often are you pumping? Is pumping comfortable for you? Are you using a nipple shield? How is that going? How much supplement is your infant eating? Is it expressed human milk or formula? Is your infant's weight being monitored by a health care provider or a WIC IBCLC?

\section{IMPLICATIONS FOR RESEARCH AND PRACTICE}

The information presented in this report could be used to enhance the breastfeeding support provided by WIC peer counselors to this population (See Table 2). Three groups of WIC peer counselors have received this education through a presentation by the first author; their responses to an informal survey indicated that the education increased their confidence in supporting these mother/infant dyads. Further research, however, is needed to evaluate the effectiveness of this education in improving maternal/infant outcomes (e.g. breastfeeding rates, mothers' breastfeeding self-efficacy, and infant hospital readmissions) and increasing the WIC peer counselors' confidence in their ability to provide information and emotional support to this population. Since conflicting information from trusted sources is a source of stress for new mothers, WIC breastfeeding staff could partner with community hospitals to develop consistent breastfeeding messages for this population. ${ }^{2,4}$ Establishing and continuing breastfeeding can be complex and difficult for mothers of late preterm infants; ongoing support in the community is essential. ${ }^{6,7,24}$ Educating WIC peer counselors about breastfeeding these infants may enhance the support they offer mothers and improve their breastfeeding outcomes. 


\section{REFERENCES}

1. Martin JA, Hamilton BE, Osterman MJK. Births in the United States, 2015. NCHS data brief, no 258. http://www.cdc.gov/nchs/data/nvsr/nvsr64/nvsr64_12.pdf. Published December 23, 2015. Accessed January 4, 2017.

2. Hackman NM, Alligood-Percoco N, Martin A et al. Reduced breastfeeding rates in firstborn late preterm and early term infants. Breastfeed Med. 2016; 11(3):119-25.

3. Wight NE. Breastfeeding the borderline (near-term) preterm infant. Pediatr Ann 2003;32(5):329-36.

4. Meier P, Patel AL, Wright $\mathrm{K}$ et al. Management of breastfeeding during and after the maternity hospitalization for late preterm infants. Clin Perinatol 2013;40(4):689-705.

5. Demirci JR, Happ MB, Bogen DL et al. Weighing worth against uncertain work: the interplay of exhaustion, ambiguity, hope and disappointment in mothers breastfeeding late preterm infants. Matern Child Nutr 2015 Jan;11(1):59-72.

6. Kair LR, Flaherman VJ, Newby KA, Colaizy TT. The experience of breastfeeding the late preterm infant: a qualitative study. Breastfeeding Med 2015;10(2):102-6.

7. Campbell LA,Wan J, Speck PM, Hartig MT. Women, Infant and Children peer counselor contact with first time breastfeeding mothers. Public Health Nurs.2014; 31(1):3-9.

8. United States Department of Agriculture [USDA] Food and Nutrition Service [FNS]. Loving Support@ Model for a successful peer counseling program. https://wicworks.fns.usda.gov/wicworks/Learning_Center/ FNS_model.pdf. No publication date. Accessed April 15, 2017.

9. Hallowell SG, Spatz DL. The relationship of brain development and breastfeeding in the late-preterm infant. J Pediatr Nurs.2012;27:154-162. 
10. Kinney HC. The near-term (late preterm) human brain and risk for periventricular leukomalacia: A review. Semin Perinatol 2006 Apr;30(2):81-88.

11. Dosani A, Hemraj J., Premji SS, Currie G, Reilly SM, Lodha AK, Young M, Hall, M. Breastfeeding the late preterm infant; experiences of mothers and perceptions of public health nurses. Int Breastfeed J 2017;12(23): s13006-017-0114-0.

12. Medoff-Cooper B, Shults J, Kaplan J. Sucking behavior of preterm infants as a predictor of developmental outcomes. J Dev Behav Pediatr 2009;30:16-22.

13. Gianni M, Bezze E, Sannino E et al. Facilitators and barriers of breastfeeding late preterm infants according to mothers’ experiences. BMC Pediatr 2016;16:179- 187.

14. Gregory KE, Samuel BS, Houghteling P et al. Influence of maternal breast milk ingestion on acquisition of the intestinal microbiome in preterm infants. Microbiome 2016; 4:68.

15. Medoff-Cooper B, Holditch D, Verklan MT et al. Newborn clinical outcomes of the AWHONN late preterm infant research-based practice project. J Obstet Gynecol Neonatal Nurs 2012;41(6):774-785.

16. Cleveland K. Feeding challenges in the late preterm infant.Neonatal Netw 2010;29:37-41.

17. Nagulesapillai T, McDonald SW, Fenton TR et al. Breastfeeding difficulties and exclusivity among late preterm and term infants: results from the all our babies study. Can J Public Health. 2013 Jul 25;104(4):e351-6.

18. Meier PP, Brown LP, Hurst NM et al. Nipple shields for preterm infants: effect on milk transfer and duration of breastfeeding. J Hum Lact 2000;16:106-114.

19. Hill PD, Aldag JC, Chatterton RT, Zinaman M. Comparison of milk output between mothers of preterm and full term infants: the first 6 weeks after birth. $J$ Hum Lact 2005;21:138-150. 
20. Post EDM, Stam G, Tromp, E. Milk production after preterm, late preterm and term delivery. J Perinatol.2015;35:1-5

21. Lucas R, Gupton S, Holditch-Davis D. et al. A case study of a late preterm infant's transition to full at-breast feedings at 4 months of age. J Hum Lact.2014 Feb;30(1):28-30.

22. Hurst NM, Meier PP,Engstrom JL, Myatt A. Mothers performing in-home measurement of milk intake during breastfeeding their preterm infants:maternal reactions and feeding outcomes. J Hum Lact 2004;20:178-187.

23. Haase B, Barreira J, Murphy P, et al. The development of an accurate test weighing technique for preterm and highrisk hospitalized infants. Breastfeed Med 2009;4:151-6.

24. Premji SS, Currie G, Reilly S, Dosani A., Oliver LM, Lodha AK, Young M. A qualitative study: mothers of late preterm infants relate their experiences of community-based care. PLoS ONE 2017 Mar;12(3):e)174419.

25. Tully KP, Holditch-Davis D, Silva S, BrandonD. Well-being among mothers of late preterm and term infants: a secondary, exploratory analysis. Adv Neonatal Care 2017;17(1):65-75.

26. Academy of Breastfeeding Medicine. ABM clinical protocol \#10: Breastfeeding the late preterm infant (340/7 to 366/7 weeks gestation). Breastfeed Med. 2011; 6(3):151156. doi:10.1089/bfm.2011.9990 
5

6

7

8

9

10

11

12

13

14

15

16

17

18

19

20

21

22

23

24

25

26

27

28

29

30

31

32

\section{Figure Legends}

Figure 1. Development of the Human Cerebral Cortex..$^{10}$

Used with permission of the copyright owner.

Figure 2. Near-term Infant (e.g. late preterm) Breastfeeding Cascade ${ }^{3}$

* Near-term Infant (e.g. late preterm)

Used with permission of Elsevier, the copyright owner. 